Du plan stratégique au plan de transport. Deux succès de logistique pour deux stratégies en échec (août 1914-septembre 1939)

August 1914-September 1939: from strategic plan to transportation plan, two logistical successes for two strategic in failure

\title{
Pierre Lepage
}

\section{CpenEdition}

\section{Journals}

Édition électronique

URL : https://journals.openedition.org/rhcf/1544

DOI : 10.4000/rhcf.1544

Éditeur

Rails \& histoire

Édition imprimée

Date de publication : 1 septembre 2012

Pagination : 109-133

ISSN : 0996-9403

Référence électronique

Pierre Lepage, «Du plan stratégique au plan de transport. Deux succès de logistique pour deux stratégies en échec (août 1914-septembre 1939) », Revue d'histoire des chemins de fer [En ligne], $42-43$ 2012, mis en ligne le 14 novembre 2014, consulté le 22 avril 2022. URL : http:// journals.openedition.org/rhcf/1544; DOI : https://doi.org/10.4000/rhcf.1544 
Pierre LEPAGE

\section{Du plan stratégique au plan de transport. Deux succès de logistique pour deux stratégies en échec (août 1914-septembre 1939)}

«La contre-offensive est la stratégie des peuples pacifiques» Jean de Pierrefeu

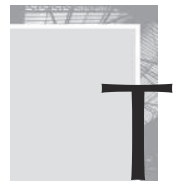

oute manœuvre stratégique implique : la surprise et, par conséquent : le secret / la vitesse de réunion des forces (troupes et logistique) ${ }^{1}$.

Au lendemain de la défaite de 1871 , les gouvernements de la $\mathrm{III}^{\mathrm{e}}$ République, l'État-major général, l'administration des Travaux publics et les compagnies de chemin de fer tirent les leçons des insuffisances et des désordres que le conflit avait mis en évidence. Sous l'impulsion de Charles de Saulces de Freycinet ${ }^{2}$, ingénieur général des Ponts et Chaussées proche collaborateur de Gambetta, qui fut ministre de la Guerre et des Travaux publics et président du Conseil à cinq reprises, une série de dispositions législatives et réglementaires constamment révisées vinrent compléter la loi initiale du 3 juillet 1877. Celleci disposait que le ministre de la Guerre prenait sous son autorité la totalité des moyens de transport en cas de crise.

1- Je remercie vivement le Service historique de la Défense qui a facilité ces travaux de recherche, le personnel des Archives de l'Assemblée nationale, très disponible, et François Formizyn pour la préparation et la réalisation informatique.

2- YVERT, 2002. 


\section{De quelques principes}

La loi du 28 décembre 1888 et ses décrets d'application successifs organisent un système dual. Le réseau ferroviaire - ou plus exactement les réseaux - étaient répartis en deux zones. Celle de l'Intérieur relevait du ministre de la Guerre, assisté par la Commission supérieure des chemins de fer, organe mixte de direction composée des directeurs des grands réseaux - les directeurs techniques - et des commissaires militaires, à savoir des officiers supérieurs attachés au $4^{e}$ Bureau de l'État-major de l'Armée. La zone des Armées était subordonnée au général commandant en chef du principal groupe d'armées (le Nord-Est), assisté d'une Commission des chemins de fer de campagne. Cette zone des armées était elle-même subdivisée en deux parties : la zone dite de l'avant, proche du front où devaient s'effectuer les opérations, serait exploitée par les compagnies de chemins de fer du Génie ( $5^{\mathrm{e}}$ régiment), susceptibles d'agir en territoire étranger en fonction des progrès de l'offensive. La seconde zone, celle de l'arrière et des étapes, dépendait d'un général, directeur des services de l'Arrière, secondé par la Direction des chemins de fer (DCF). À ce niveau, des commissions mixtes de réseaux exerçaient leur activité sur toutes les voies ferrées du réseau des armées. À l'approche de la guerre, la mission des chemins de fer fut rendue encore plus précise par l'article 18 et son annexe 8 au décret du 2 décembre 1913 relatif au service des armées en campagne ${ }^{3}$.

Le passage du pied de paix au pied de guerre impliquait l'élaboration d'un " plan stratégique " conçu de longue date par l'État-major général. Un plan stratégique englobait plusieurs sous-ensembles articulés et mis en mouvement comme par une sorte de machinerie complexe : plan de mobilisation, plan de couverture, plan de concentration, plan de sûreté, plan de transport, plan de manœuvre. Tous ces plans étaient plus ou moins partagés avec le gouvernement en charge de la conduite politique de la guerre. Au sommet était le "plan d'opérations ", le plus secret, qui relevait de la réflexion et de l'autorité exclusive du général commandant en chef. Entre 1871 et 1914, l'État-major général prépara 18 plans successifs dont l'un ne reçut pas de numérotation. Le dernier plan mis au point en 1913 fut dénommé «Plan XVII ».

Pour adopter la formulation des mathématiciens modernes, disons qu'un plan stratégique peut être considéré comme un espace vectoriel de dimension trois : les moyens, l'espace et le temps. Les moyens à " projeter " sur les frontières, pour adopter le langage des militaires, sont faits alors pour

3- Voir Service historique de la défense (désormais : SHD), Collection Campet de Saujon. Ordonnances, lois, décrets militaires. Série 1X. 
l'essentiel de matériel humain, 800000 hommes appuyés par le canon de 75 et par un contingent initial de 200000 chevaux. Ces effectifs sont répartis entre 22 corps d'armée fournis par les régions militaires et forment un total de 46 divisions d'active, auxquelles s'ajoutent 5 groupes de divisions de réserve, soit 22 divisions.

L'espace assigné aux opérations est déterminé par des considérations diplomatiques et par des accords secrets conclus avec les puissances alliées et les neutres. Nous y reviendrons brièvement pour autant que ces causes aient une influence sur l'emploi des réseaux ferrés.

Le temps est le facteur primordial pour les militaires. À l'époque, le temps est régulé par le chemin de fer, par son potentiel, par sa capacité à assumer le plus rapidement possible l'exécution de la manœuvre stratégique. L'examen attentif des procès-verbaux du Conseil supérieur de la Guerre (désormais : CSG $)^{4}$ traduit l'obsession des généraux suscitée par l'extension du réseau des chemins de fer allemands en Rhénanie. Le développement d'un important réseau ferroviaire entre Trêves et Aix-la-Chapelle, orienté vers la Belgique, répond à la stratégie originale du général comte Schlieffen d'enveloppement du dispositif français par la violation du territoire neutre belge. Les prévisions de l'état-major français tablent alors sur un délai de onze jours pour la concentration des corps actifs allemands. Dans l'esprit de nos stratèges, hantés par une "attaque brusquée ", la nécessité de gagner l'ennemi de vitesse, à tout le moins d'égaler ce délai, implique une amélioration conséquente des réseaux du Nord et de l'Est (fig. 1).

La vitesse, nous le savons, est la dérivée de l'espace par rapport au temps. Or il convient de revenir un moment sur cet espace concédé aux militaires par le pouvoir politique. D'une part, en juillet 1911 des différends et une crise du haut commandement se sont produits à propos de la conception du plan stratégique. Le général Michel, alors vice-président du CSG et généralissime désigné, a perçu très tôt les intentions d'une offensive ennemie de grande ampleur en Belgique, "cette terre historique des grandes invasions " point de vue, la bataille générale et décisive doit être livrée au cœur de ce pays. De plus, pour garantir l'équilibre des forces en présence, Michel propose de réunir sous forme de demi-brigade chaque régiment d'active et son régiment de réserve. Avec un effectif ainsi doublé, il propose une répartition entre deux

4- Voir les procès-verbaux des séances du Conseil supérieur de la Guerre de 1908 à 1914.

5- Les Armées françaises pendant la Grande Guerre, Paris, Imprimerie nationale, 1922-1937, 106 volumes, dont 24 de texte (Précis), 56 d'annexes et 26 de cartes, vol. 1, annexes et cartes, «Le plan Michel ». 


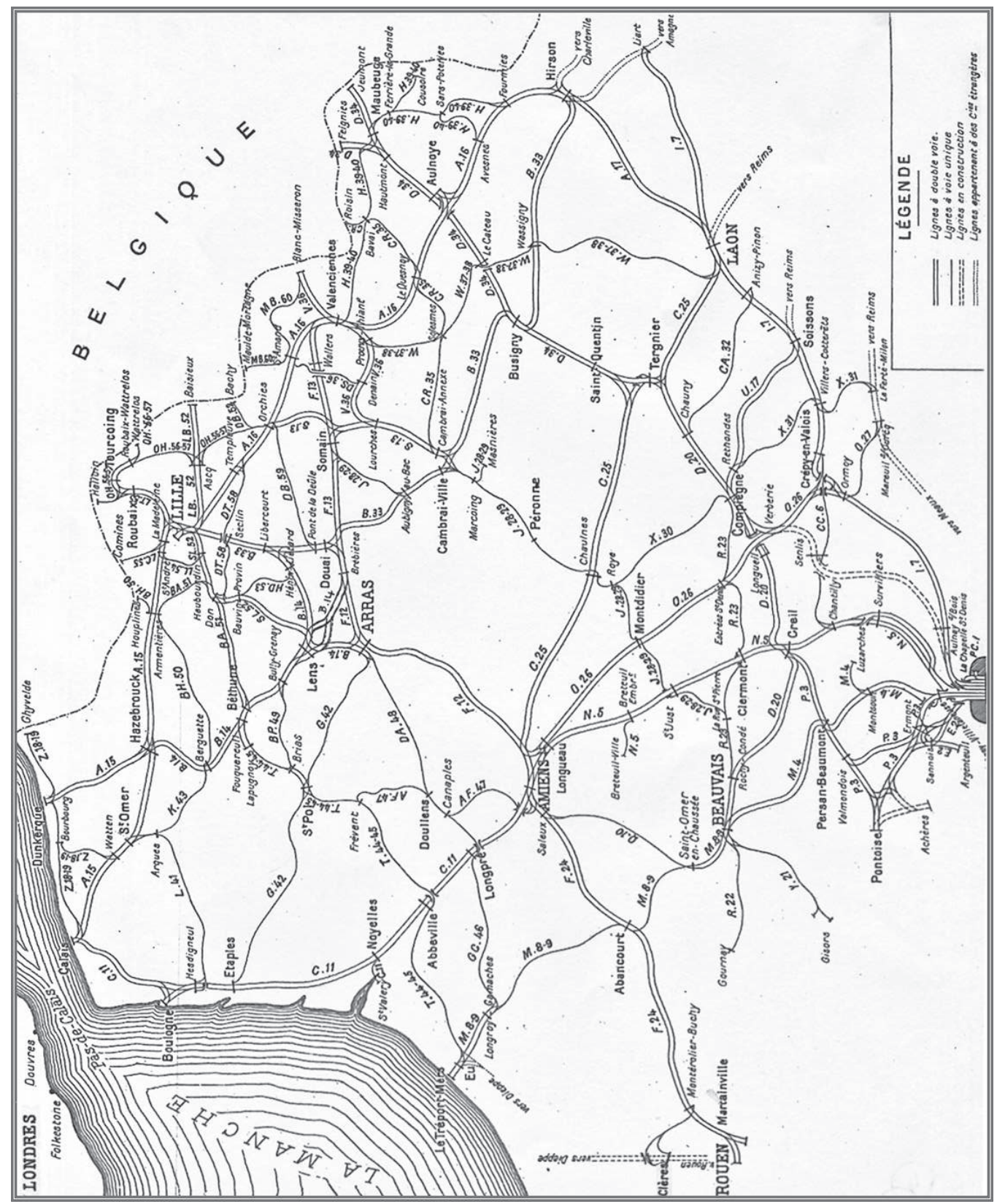

Figure 1. Carte du réseau Nord. Cote SHD 16N2813. 
groupes d'armées, l'un pour deux tiers sur la frontière du Nord de Dunkerque à Maubeuge, le troisième tiers couvrant la frontière de Belfort jusqu'au système fortifié des Hauts de Meuse. On observera que la distance à parcourir pour border la frontière du Nord est moindre, de cent kilomètres, que celle gardée par les systèmes fortifiés de l'Est, d'où un gain de temps non négligeable. Mais le réseau du Nord était-il en capacité d'exécuter un plan de transport d'une masse d'hommes passant par cette stratégie de 800000 à 1700000 ? Certes le réseau du Nord, traversant un plat pays, était réputé pour la densité de ses lignes et pour la célérité de ses machines de traction. Était-ce suffisant ? Le général Michel suggérait un substantiel effort d'investissement que le président de la Compagnie du Nord, le baron Edouard de Rothschild, ne semblait guère disposé à consentir. Michel mis en minorité et évincé, son successeur le général Joffre songeait tout autant à une offensive générale en Belgique. Il en fut dissuadé lors d'une " réunion secrète » qui se tint le 21 février 1912 au ministère des Affaires étrangères ${ }^{6}$. Pour des raisons diplomatiques, le président du Conseil, Raymond Poincaré, imposa au chef d'État-major général Joffre une offensive orientée vers la Lorraine, à la rigueur dirigée vers le Luxembourg. Le réseau de l'Est devrait donc porter presque tout le poids du plan de transport. À cela s'ajoutaient toutes les expectatives nées de l'attitude équivoque de l'Italie, qui avait certes promis sa neutralité par un accord secret conclu en 1902 avec Delcassé. Cet accord fut longtemps ignoré des militaires français, lesquels maintenaient deux corps d'armée en permanence sur les Alpes. Telle était la problématique à laquelle était confronté l'État-major français en 1914.

À travers toutes les bases du Plan XVII, bornons-nous à examiner quelques aspects du «plan de transport » mis en œuvre à l'été de 1914.

\section{Le plan de transport. Ses moyens. Son exécution}

Que comporte un plan de transport ? Les itinéraires et les marches correspondantes, les moyens d'embarquement et de débarquement des troupes, des animaux, des matériels et équipements, les stations haltes d'abreuvage et de ravitaillement, l'emplacement des postes de régulation, les équipes mobiles de réparations, un réseau téléphonique spécialisé de communications.

Le rassemblement dans les zones de concentration des armées mobilisées sur l'ensemble du territoire national nécessite l'organisation de plusieurs catégories de transport dont les principales visent la mobilisation, la couverture

6- Sur cette réunion secrète voir les procès-verbaux du conseil supérieur de la défense nationale et PÉDRONCINI, 1977. 
et la concentration. En 1914, seule la voie ferrée à l'écartement normal est apte à assumer cette mission complexe. François Caron a ainsi recensé avec précision le nombre de trains mis en route pour chacune des catégories de transport ${ }^{7}$.

La mobilisation génère les déplacements de groupes d'hommes ou d'isolés qui rejoignent leur centre mobilisateur. Ils s'effectuent, dès le 2 août, pendant les quatre premiers jours suivant la publication du décret de mobilisation, soit par les trains du service commercial, soit par des trains spéciaux. Près de 10000 trains ont concerné « la mobilisation qui n'est pas la guerre ", suivant la célèbre proclamation du gouvernement, mais qui en est un sérieux avant-goût. «Dans l'enthousiasme les agents de la Compagnie du Midi montrent beaucoup de zèle et de bonne volonté ${ }^{8}$ ", note par exemple Giboudot, le commissaire militaire de ce réseau.

Parallèlement s'exécutent vers la frontière les transports de couverture. Ils anticipent et accompagnent la mobilisation. À partir du 31 juillet commence le transport des six corps d'armée frontaliers. Les hommes étant "sur place ", c'est-à-dire cantonnés dans les garnisons de l'Est, à l'effectif de guerre, les distances à parcourir sont courtes. La couverture exige la mise en route de 573 trains de combattants (TC), dont près de 300 sont fournis par le seul réseau de l'Est.

Le transport de concentration vers la frontière dans le délai $\mathrm{J}+11$, des 16 autres corps actifs et, trois jours plus tard, des 5 groupes de divisions de réserve, constitue la pièce maitresse du Plan. Suivent alors les parcs d'artillerie, les convois, les équipages de ponts... La première phase a demandé 2534 trains, la seconde phase 2744 convois. Le Plan a fonctionné suivant les prévisions. Au soir du 18 août la concentration est achevée. Les grandes opérations peuvent débuter en Lorraine.

Dans une vision napoléonienne, les stratèges souhaitaient utiliser le chemin de fer «à bloc » pour "sauter à la gorge de l'ennemi ", avec la conviction que « l'offensive poussée jusqu'à l'excès est la meilleure des sûretés, quelle que soit par ailleurs l'indigence de la conception " (colonel de Grandmaison"). Mais la capacité du réseau de l'Est touchait à son point de saturation. Il n'était pas possible " de tracer un courant de plus ", alors que le Nord offrait encore d'excellentes possibilités.

7- CARON, 2005, p. 540 et suiv.

8- SHD, 7N2043.

9- GRANDMAISON, 1911. 
Dans le même temps, plusieurs décrets pris en conseil des ministres renforçaient ce que le général André Bach dénomme " la dictature de Joffre » : proclamation de l'état de siège sur l'ensemble des départements métropolitains et de l'Algérie, en vertu d'une loi, sorte de succédané de celle du 9 août 1849, qui demeura plus de cent ans en vigueur, subordination des préfets de la zone des armées à l'autorité militaire, instauration de la censure, suspension du service commercial des chemins de fer, mise sous la tutelle du haut commandement de l'intégralité du réseau de l'Est et de quelques lignes du Nord et du PLM...

Pour acheminer les troupes vers leurs zones de concentration, la direction des Chemins de fer disposait de dix lignes de communications soigneusement tracées par l'État-major depuis de nombreuses années. Nous avons décrit le rôle de ces lignes dans un précédent article ${ }^{10}$. Nous ne leur accordons ici qu'un bref rappel. Chacun des itinéraires de ligne aboutit à une gare régulatrice, siège d'une commission régulatrice mixte, avec son commissaire technique et son commissaire militaire. Dans la zone d'action de cette régulatrice, des lignes orientent les troupes vers des gares de rassemblement et de débarquement équipées de quais militaires. Enfin, des gares de ravitaillement complètent l'ensemble. Des commissions mixtes de gares administrent le trafic à chaque échelon d'une hiérarchie à la fois complexe, souple et rigoureuse.

Toutes les unités d'un même corps d'armée sont fractionnées en éléments de transport correspondant chacun à la capacité d'un train militaire et suivent la même ligne de communications. Chaque train, d'une longueur totale de 350 mètres, comprend 50 wagons PV (petite vitesse), les célèbres " hommes 40, chevaux en long 8 ». Ils sont équipés de bancs mobiles. La composition des rames est invariable : elles sont interchangeables. Chaque train circule à la vitesse de $30 \mathrm{~km} /$ heure en fonction d'un tableau de marche et d'un ordre de circulation remis au responsable de convoi avant le départ. La destination est maintenue secrète jusqu'à la gare régulatrice. Le transport d'un corps d'armée avec son état-major, ses services, son artillerie, sa cavalerie, nécessite de 60 à plus de 100 trains, suivant que ce corps est composé de deux ou trois divisions. Le tableau horaire de chaque ligne est prévu pour 48 marches journalières, soit un train toutes les 30 minutes, programme extensible jusqu'à 56 marches. À cet horaire rigoureux, les technocrates du $4^{\mathrm{e}}$ bureau ont intégré les constantes de chantiers, c'est-à-dire le temps nécessaire pour l'embarquement et le débarquement des troupes et des animaux, le chargement et le déchargement du matériel. Les constantes sont déterminées par les ressources

10- LEPAGE,1996. 
des gares en nombre de voies, en quais latéraux ou en bout, en matériels de levage, etc. ${ }^{11} \mathrm{Au}$ total, la puissance du réseau français est de 480/24 marches, celle du réseau allemand de 720/24. Côté français, il existe vers l'AlsaceLorraine 4 pénétrantes et 16 liaisons depuis l'Allemagne.

L'espacement entre les trains est régulé suivant deux principes : l'espacement par le temps, avec marche à vue, l'espacement par la distance, avec le block-system et par l'application stricte du code des signaux de 1885, qui a conduit à une relative harmonisation des feux et panneaux et de leurs couleurs, tout en laissant subsister des particularités sur les différents réseaux. Lélectro-sémaphore Lartigue est généralisé sur l'Est, mais les militaires privilégient le cantonnement téléphonique à block non enclenché ${ }^{12}$, ce qui fut la cause de plusieurs accidents meurtriers, avant même les premiers combats, que la censure s'empressa de masquer ${ }^{13} \ldots$

On voit donc que la densité du trafic, le rendement de chaque ligne, sont privilégiés par rapport à la vitesse des convois. Dans ces conditions, les prestigieuses Pacific ne jouent pas un rôle de premier plan, hormis la rapidité de déplacement de quelques hommes politiques et d'états-majors de haut niveau. Le gain de vitesse est recherché par d'autres moyens.

La concentration achevée, il fallait assurer le ravitaillement des armées en munitions, en matériels, en vivres, harnachements, habillements, etc. Dans cette guerre de masse, caractérisée par les constantes modifications adaptées aux exigences de la manœuvre stratégique imposée par le $3^{\mathrm{e}}$ Bureau chargé des opérations, il fallait au chemin de fer un organe de direction souple, capable de donner aux réseaux une forte réactivité. Les lignes de communications à deux voies qui apportent les ressources du territoire national et « qui recueillent les déchets " (c'est-à-dire les éclopés), étaient trop rigides pour répondre aux brusques déplacements des forces opérationnelles. Elles furent complétées par des liaisons transversales, des "rocades ", constituant le second réseau de communications. Des tracés et des graphes avaient été étudiés dès l'avant-guerre, non sans difficulté. Les rares rocades disponibles étaient situées loin du front. De plus, la convergence des réseaux vers la capitale provoquait un trafic intense sur les ceintures et leurs points d'échanges (Noisy, Epluches, Achères, Juvisy).

Rendons compte brièvement de quelques dispositions qui avaient été prises pour augmenter la vitesse des déplacements :

11- SHD, 7N2042 à 2045.

12-GERNIGON, 1999, p. 142, 156 et 163-164.

13- SHD, 16 N2863. 
- Matériel et exploitation : usage intensif du matériel roulant par la composition des rames interchangeables et l'emploi important des wagons tirés du parc des 380000 unités des compagnies ferroviaires. Adaptation des quais à la composition de ces rames. Mise en réserve de centaines de locomotives.

- Infrastructure : création de nombreux raccordements directs pour éviter les rebroussements en gare, mise au gabarit de nombreux tunnels, évitements, tracé d'itinéraires de détournement hors de portée de la grosse artillerie, comme au sud de Nancy, création et extension des faisceaux de triage (Trappes, Blaye). Amélioration des liaisons entre le réseau des Armées et celui de l'Intérieur et des points de jonction entre les réseaux voisins (Is-sur-Tille). Raccordements ferroviaires aux ports maritimes et fluviaux, aux établissements militaires, aux arsenaux, aux établissements privés. On note la création d'une nouvelle ligne à deux voies entre Motteville, Clères et Serqueux facilitant la pénétration entre les réseaux de l'État et du Nord. La construction d'une grande estacade à Châlons-sur-Marne permet de faire franchir la Marne et la grande voie ferrée Paris-Avricourt à tout un corps d'armée, sans cisaillement des voies.

Mais l'une des opérations les plus réussies pour relever la droite d'asymptote des $30 \mathrm{~km} /$ heure concerne le réseau de l'Est, par le quadruplement de la ligne de Paris à Avricourt entre Vitry-le- François et Nançois-Tronville jusqu'au pied des Côtes de Meuse. Ce quadruplement, réalisé avant 1914, permet l'acheminement de deux corps d'armée " en parallèle " et non plus en ligne de file ou enchevêtrés. Cette réalisation se complète par le redressement de longues courbes et par la construction des "sauts-de-mouton " de Blesme-Haussignemont, Revigny et Nançois-Tronville. L'ouvrage de Revigny combiné avec celui de Mussey, près de Bar-le-Duc, est le plus spectaculaire. Trois corps d'armée peuvent y transiter en courants totalement indépendants orientés d'Est en Ouest et du Nord au Sud. On peut en voir encore de nombreux vestiges en parcourant la " ligne classique " entre Paris et Bar-le-Duc. Il est vrai que ces réalisations ont durablement transformé le paysage. Quand on songe que Revigny porta longtemps le nom bucolique de Revigny-auxVaches ${ }^{14}$, on imagine la somme des transformations entreprises pour satisfaire les besoins des états-majors. Comme l'écrivit Jean de Pierrefeu, moins pourtant que les centaines de milliers de valeureux poilus, les logisticiens $d u 4^{e}$ Bureau « ont gagné la victoire autant que les stratèges du $3^{\mathrm{e}} »^{15}$.

14- Archives départementales de la Meuse, 40 S 1 à S $8 ; 40$ S $507 ; 56$ S 1.

15- PIERREFEU, 1922. 
Les compagnies du Génie, les sections de chemin de fer de campagne, des groupes de prisonniers ont procédé à la pose de 400 kilomètres de voies, de 900 appareils, remué plus de 700000 mètres cube de terre (fig. 2). Les compagnies de chemin de fer, fortes d'un effectif de 307000 cheminots, en ont placé près de 40000 en affectation spéciale, munis d'un brassard, à la disposition absolue de l'autorité militaire ; près de 10000 femmes ont été embauchées pour suppléer les absences ${ }^{16}$, en participant à la gestion d'un trafic dont le tonnage transporté dépassa bientôt de $40 \%$ celui du début de l'année 1914 (fig. 3).

En cette année-là, seule la voie ferrée à écartement normal était en mesure d'exécuter le plan de transport préparé par le $4^{\mathrm{e}}$ Bureau de l'État-major général. Le plan s'avéra un grand succès logistique, malgré une vingtaine d'accidents, déraillements, prises en écharpe, tamponnements, dans le contexte d'engagement sur l'Est et le Nord d'équipes de conduite des autre réseaux, formées à la hâte et peu familiarisées avec des signalisations spécifiques. Ce succès suscita l'optimisme du Grand Quartier Général de Vitry-le-François, au point que l'aide major général Berthelot déclara avec emphase : "Les acteurs sont en place. La parole est aux exécutants. " Les défaites aux frontières devaient démentir cet excès de confiance, mais elles appartiennent à d'autres fragments de l'histoire.

Terminons sur ce chapitre par une note humoristique. En 1911, le commandant Driant, député de Nancy, qui critiquait la nomination du général Joffre au poste suprême de l'armée, écrivait : " Joffre ? Il ressemble à un chef de gare!» Quand on connaît la maîtrise avec laquelle le commandant en chef sut exploiter ses réseaux dans un contexte tragique, on se dit que ce commentaire acerbe ne manquait pas d'à-propos.

\section{9-1939 : d'une guerre à l'autre}

Au lendemain de la Grande Guerre, les réseaux ferroviaires français sont à bout de souffle. Le réseau de l'Est, très éprouvé, a subi des destructions considérables. Le réseau du Nord est exsangue. L'ennemi l'a détruit méthodiquement lors de ses replis en 1917 et 1918. Les voies ont été arrachées, «à la charrue », sur de grandes longueurs ${ }^{17}$. En contrepartie, aux termes de l'Armistice du 11 novembre 1918, la France récupère l'intégralité du réseau d'AlsaceLorraine en bon état relatif, ainsi que du matériel roulant de l'État prussien

16- Certaines ont même été frappées à coups de canne par des officiers - traduits en Conseil de guerre - pour avoir fait appliquer strictement la règlementation sur la fermeture des passages à niveau, SHD, $16 \mathrm{~N} 2815$.

17- LE HÉNAFF, 1922a. 


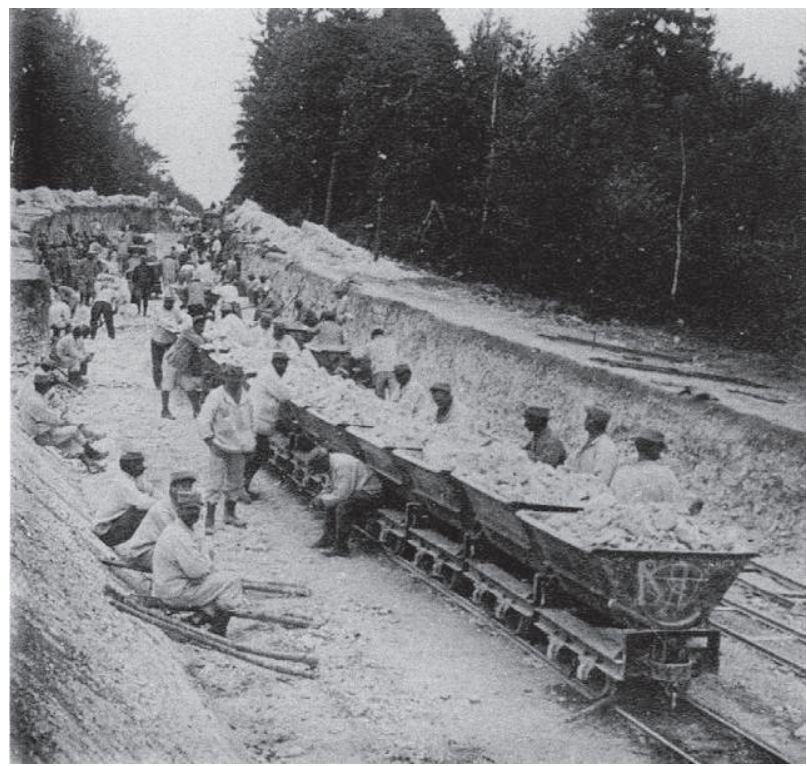

Figure 2. Un chantier de voie non localisé pendant la Première Guerre mondiale. Cl. Coll. P. Lepage.

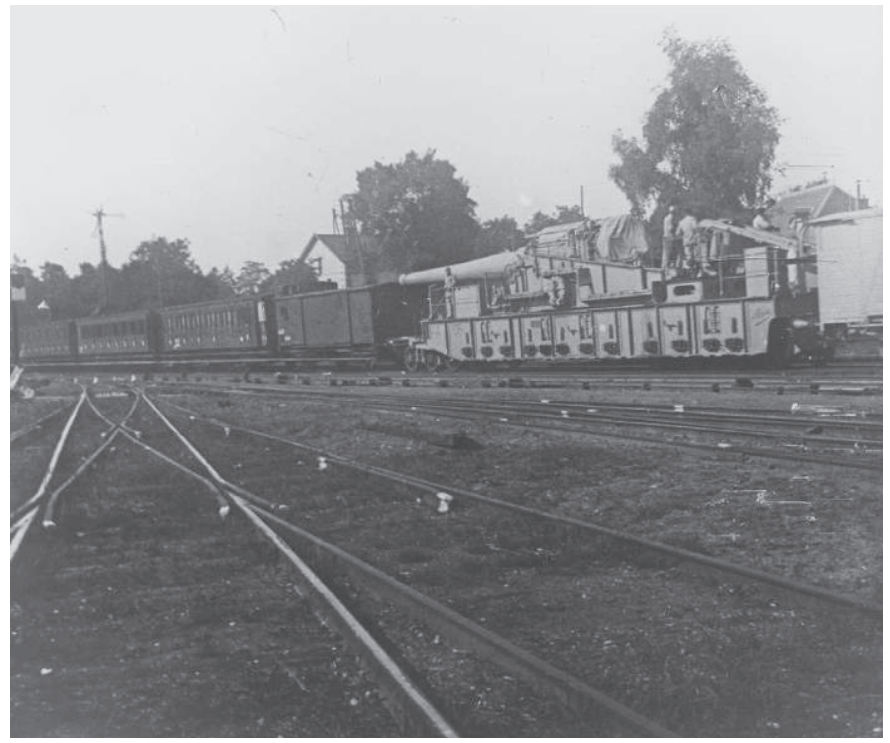

Figure 3. Nouvelle pièce d'artillerie de 320, attelée à un train en stationnement dans une gare de Picardie. Le sémaphore est effectivement du type réseau Nord. Cl. Coll. P. Lepage. 
en quantité non négligeable, même des wagons en béton armé ! Les années 1920 sont celles de la reconstruction dont les étapes ne relèvent pas de notre propos. Au Conseil national économique, Dautry exige de l'efficience : «Il faut s'abstraire de tout échange d'idées superflues qui ne puisse pas conduire à un résultat ${ }^{18}$."

Au sein de cet organisme, comme au Conseil supérieur de la défense nationale, les experts militaires mandatés par l'État-major général veillent avec une vigilance toute particulière aux impératifs de ce qu'il est convenu d'appeler désormais la Défense nationale de préférence à la Guerre. Ils s'opposent notamment à l'électrification des chemins de fer du Nord et de l'Est, en raison, affirment-ils, de la vulnérabilité des installations électriques aux bombardements aériens.

Revenons un instant sur la nouvelle dénomination du ministère de la Guerre en usage jusqu'alors. Un premier ministère dit de « la défense nationale » apparaît en février 1932, dans le troisième gouvernement Tardieu. Cette nuance est loin d'être anodine. Car enfin les gouvernements pusillanimes de cette époque, qui succèdent aux " gouvernements guerriers » des années antérieures, ne préconisent-ils pas une politique exclusive de défense nationale, de préférence à une stratégie offensive dont ils se seraient donné les moyens face à la montée du péril hitlérien ? Par la suite, Edouard Daladier transigera entre ces deux conceptions en prenant le titre de «ministre de la Défense nationale et de la Guerre ».

Il faut comprendre que, dans la première option stratégique finalement retenue, le système fortifié français s'est déplacé de 150 kilomètres à l'Est, en quittant les Hauts de Meuse pour la ligne Maginot, dont les principaux ouvrages, gardés sur le Rhin, sont implantés au voisinage de la frontière, de l'Alsace jusqu'à Montmédy. Par ailleurs, l'attitude belliqueuse de l'Italie de Mussolini oblige le Haut commandement français à maintenir en permanence deux corps d'armée sur la frontière des Alpes et dans les intervalles entre les modernes ouvrages de la ligne Maginot du secteur fortifié des AlpesMaritimes, pour la défense duquel le chemin de fer jouera un rôle important en juin 1940. Telles sont les données d'un problème auquel l'État-major général se doit d'apporter des solutions en 1938-1939, sans perdre de vue l'impératif de la célérité du déplacement des unités sur les différents fronts.

18- SHD, $2 \mathrm{~N} 148$. 
À l'aube du second conflit mondial, les principes établis avant 1914 subsistent dans le domaine législatif et réglementaire, compte tenu de l'expérience acquise. Les lois du 3 juillet 1877 et du 28 décembre 1888 restent en vigueur et fondent des décrets successifs. Les deux zones distinctes - armées et intérieur - sont redéfinies. La vieille loi du 8 juin 1849 ordonnant la mise en état de siège de l'ensemble des départements pourra être réactivée, et elle le sera effectivement le 4 septembre 1939, de même que la suspension des services commerciaux. Mais il faut explorer le maquis des textes et des décretslois publiés par le gouvernement Daladier - dans lequel Dautry est ministre de l'Armement - pour appréhender les dispositions essentielles concernant la mobilisation du réseau ferroviaire. Citons notamment :

- La loi du 11 juillet 1938 portant sur « L’organisation générale de la nation en temps de guerre ». Elle fixe le cadre très général d'un dispositif adapté aux circonstances.

- Le décret du 25 novembre 1938 relatif à la réquisition des voies ferrées exploitées par la SNCF.

- Le décret du 28 novembre 1938, portant mise en état de réquisition des agents et ouvriers des services publics de l'État, des départements, des communes et des services concédés.

Au crédit du gouvernement Daladier, mentionnons enfin le décret du 29 juillet 1939 relatif au programme de travaux de premier établissement de la SNCF. Ce programme prévisionnel, d'un montant de quatre milliards de francs, inclut « des travaux d'électrification d'une rentabilité certainement élevée et hautement utiles à l'économie du pays ${ }^{19}$. Étendu sur une période de cinq années, de 1940 à 1944, ce plan ambitieux ne pourra être mené à bien qu'après la guerre.

François Caron observe que « la nationalisation du chemin de fer était seule susceptible de libérer les énergies étouffées en rendant la tutelle [de l'État] moins pesante et moins soupçonneuse ».

Il est vrai que, d'un point de vue stratégique, la création de la SNCF au $1^{\text {er }}$ janvier 1938 renforce d'une nouvelle manière la cohésion entre l'administration et les autorités militaires grâce à la rationalisation des structures, des organisations et des méthodes de gestion. Le rattachement du réseau d'Alsace-Lorraine, géré jusqu'ici par l'État, à la nouvelle région de l'Est donne à l'État-major général plus de souplesse pour élaborer son plan stratégique et de transport.

19- Journal officiel, 30 juillet 1939. 
La Commission supérieure militaire des chemins de fer est réorganisée. Les officiers supérieurs et brevetés du $4^{\mathrm{e}}$ Bureau y siègent aux côtés des hauts dirigeants de la SNCF, sous la présidence du chef d'État-major général, Gamelin. On note (enfin !) l'entrée dans cette commission d'un officier supérieur représentant la jeune Armée de l'Air (décret du 4 février 1939). Les décisions de cette Commission supérieure qui, au cours des opérations de guerre, se transforme en "Commission centrale ", sont relayées au niveau des cinq régions de la SNCF par des commissions mixtes, des commissions régulatrices et des commissions de gares, l'ensemble fonctionnant suivant les principes de 1914-1918. La direction des chemins de fer, rattachée au GQG, est placée sous l'autorité du colonel, puis général Kergoat. Il convient également de souligner un indéniable effort de formation et de perfectionnement entrepris dès 1932 en direction des officiers chargés de l'exploitation des chemins de fer en temps de guerre. Le $4^{e}$ Bureau organise des sessions de formation d'officiers régulateurs de concentration ou de mouvements. Les cours magistraux et les exercices sur la carte sont complétés par des visites sur le terrain, par la reconnaissance des installations. Chaque stagiaire est doté d'un Mémento très documenté et reçoit périodiquement le Bulletin mensuel de l'École de perfectionnement des officiers de réserve du service des chemins de fer, qui fait l'objet d'une publication de 1932 à $1939^{20}$ (fig. 4). En septembre 1938, avant que ne s'ouvrent « les négociations de Munich ", le rappel de " certaines classes de réservistes " permet de tester par un exercice en temps réel l'efficacité du plan de transport, sousensemble du Plan stratégique E et du Plan W concernant le corps expéditionnaire britannique. Le rappel de ces troupes, s'il " ne constitue pas une mobilisation " aux dires du gouvernement français, se situe encore moins dans un état d'esprit de guerre et masque en fait le prélude du désastre diplomatique de Munich.

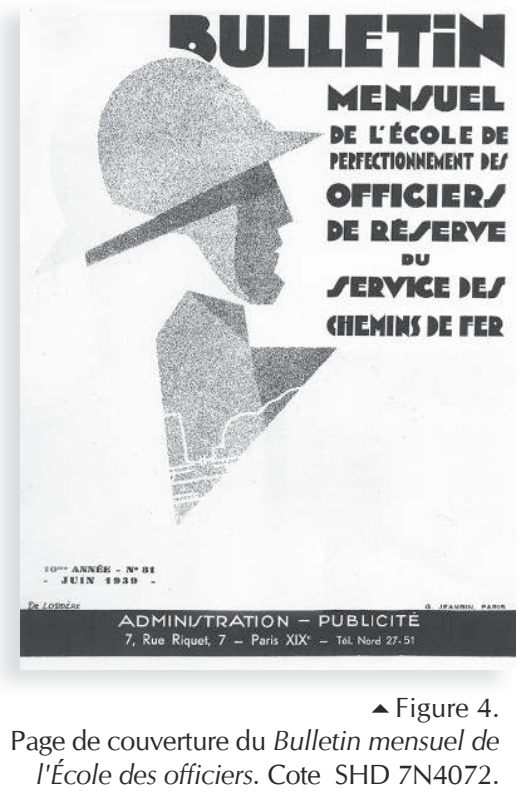

\footnotetext{
20- SHD 7N4072, série 1919-1939. Sur cet article, ainsi que sur le vécu des événements sur le terrain en mai et juin 1940, on lira avec profit les Souvenirs du capitaine Robida, officier régulateur à la Direction des chemins de fer.
} 
Sur le plan technique, le chemin de fer bénéficie pour accomplir la mission qui lui est assignée de l'expérience et des acquis du conflit précédent, mais également des innovations et des progrès qui, pourtant, ne lui donnent guère l'occasion de disposer de ce "supplément de vitesse » réclamé par l'État-major, dont la situation des anciens réseaux ne lui offrait pas l'opportunité. Un relevé effectué par le $4^{e}$ Bureau donne une vitesse moyenne de 27,6 km/heure sur les six rocades de ravitaillement. Il est vrai que la problématique des transports spéciaux (chars, essence, etc.) entraîne une plus grande complexité qu'en 1914. À la rationalisation des méthodes répond une amélioration notable des itinéraires stratégiques entre les 20 régions de corps d'armée et leurs gares régulatrices. Elle est obtenue par une rectification des tracés, par le doublement de certaines sections de lignes à voie unique et la suppression de rebroussements, par le renforcement des infrastructures, la consolidation des ouvrages d'art, l'emploi de rails plus lourds et de traverses métalliques, etc. L'adoption du Code Verlant en 1934, le développement des signaux unifiés et de la signalisation lumineuse commencent à porter leurs fruits dans le domaine de l'augmentation de la densité et de la vitesse des convois sur les itinéraires réservés à la concentration. L'électrification achevée en 1937 et 1938 sur l'Ouest (Paris-Le Mans), le Sud-Ouest (Paris Bordeaux) et sur la Grande Ceinture sud de Paris contribue aussi à produire plus de vitesse par l'effet de la réduction du nombre de relais de traction.

C'est essentiellement sur la région de l'Est, à la requête de la Commission de défense des frontières exprimée dans sa séance du 3 juillet 1922, et de l'État-major, que sont réalisés les grands et spectaculaires travaux d'infrastructure. Le projet de quadruplement intégral de l'itinéraire Paris-Strasbourg est rendu effectif entre Vitry-le François et Lérouville par l'extension de la section déjà réalisée jusqu’à Nançois (fig. $5 \mathrm{a}$ et $5 \mathrm{~b}$ ). Notons que les militaires sont réticents devant ces liaisons doubles, exposées aux bombardements aériens. Toutefois, cette réalisation permet de franchir plus aisément les dures rampes des Côtes de Meuse avec l'emploi de locomotives plus puissantes et plus véloces. Cet ensemble est remarquablement complété par la création d'une liaison directe et rectiligne par le Rupt de Mad, affluent de la Moselle, apte aux grandes vitesses, entre Lérouville et Metz, équipée de la signalisation lumineuse d'approche. L'accomplissement est obtenu avec la construction des grands sauts-de-mouton de Metz-Frescaty, Waville, Arnaville, Novéant et Lérouville, celui-ci complété par un raccordement direct entre les axes ParisNancy et Paris-Metz. L'exécution d'un ouvrage similaire à Frouard, en avant de Nancy, met un double itinéraire vers Metz par la vallée de la Moselle à 
Du plan stratégique au plan de transport

Figure 5a

Page de couverture du Projet d'exécution des travaux d'infrastructure. Cote SHD 7N4071.

- Figure 5b. Schéma du dispositif des voies entre Vitryle-François et Lérouville. Cote SHD 7N4071.

SERVICE DE LA VOIE ET DES TRAVAUX

Arrønđtiasioment a da Fravaux
DÉpARTEMEXY DE LA MEUSE

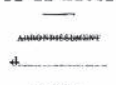
$-15^{-3}-$

COMPAGNIE DES CHEMINS DE FER DE LEST

ACHËVEMENT DE LA LIGNE

DE VITRY-LE-FRANGOIS A LËROUVILLE

PROJET D'EXECUTION DES TRAVAUX DINFRASTRUCTURE

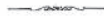

Souts de mouton de Sézouvilk.<smiles>CCC(C)[Ge]</smiles>
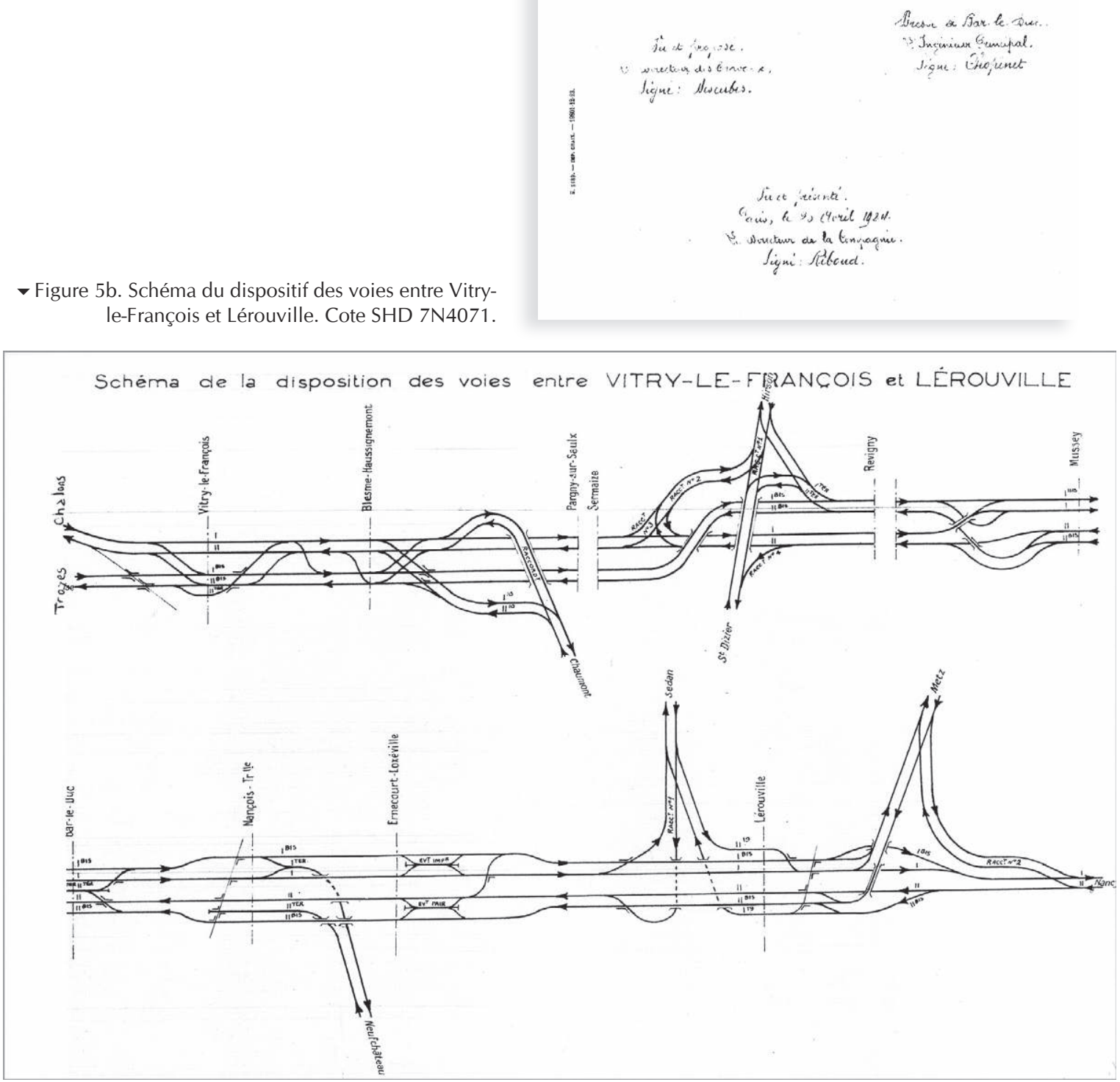
la disposition du haut commandement ${ }^{21}$. L'extension de la ligne de NeuvesMaisons à Blainville améliore le contournement de Nancy. Le dispositif se complète par un projet inachevé de " percée des Vosges " (Saint-Dié - Saales, tunnel de Sainte-Marie-aux-Mines).

Le réseau d'Alsace-Lorraine avait été conçu par les Allemands comme orienté vers la France. Après 1918, les stratèges et les logisticiens l'aménagent en direction de la ligne Maginot, pour ne pas dire de l'Allemagne. Les grands sauts-de-mouton de Novéant près de Metz, de Xouaxange, non loin de Sarrebourg, facilitent l'interversion des sens de circulation entre les deux réseaux, la circulation à droite "à l'allemande » étant maintenue en AlsaceLorraine pour réduire le coût de l'investissement. La signalisation spécifique est adaptée progressivement au Code Verlant. Ce principe sera repris beaucoup plus tard pour le raccordement à Baudrecourt de l'axe Metz-Strasbourg à la LGV Est. Tous ces grands travaux réalisés entre les deux guerres ont modifié profondément les paysages et ces ouvrages subsistent comme les témoins d'une époque révolue.

Au total, le plan de transport militaire de 1939 a permis l'acheminement des troupes françaises sinon dans l'enthousiasme, du moins dans des conditions de confort plus satisfaisantes qu'en 1914. Les soldats ont, plus souvent, voyagé dans des voitures certes de $3^{\text {e }}$ classe, moins éprouvantes cependant que les antiques wagons PV de la mobilisation précédente. Par ailleurs, il avait fallu concevoir, après le remplacement d'une partie de la cavalerie, des wagons remorques agencés pour le transport des 45 bataillons de chars de combat, ces Somua de 20 tonnes, groupés non pas en masses compactes, mais répartis entre les divisions d'infanterie, ce qui fut, selon les experts, à l'origine de la défaite en juin 1940. Pour ces engins, il fallait de l'essence, acheminée dans des citernes à vin transformées, par des itinéraires spécifiques. On lit, dans les Souvenirs de Robida, cet exploit qui consista à « livrer " par le chemin de fer les chars de combat au colonel de Gaulle pour sa contre-attaque sur Montcornet où cent blindés allemands faisaient tête.

En 1939, l'effort militaire français fut sensiblement équivalent à celui consenti en 1914. Un dénombrement effectué en mars 1940 met cependant en évidence le fait que les effectifs de l'armée de terre étaient inférieurs de 415000 hommes à ceux de mai 1917. Si l'on considère que «la concurrence " disposait de 188 compagnies d'auto transport, il semble loisible de dire que le plan de transport par fer en fut facilité. Mais une fois retranchées derrière la

21- Archives départementales de la Moselle, 7S19 60 ; 65-66; 68 et S3. 
ligne Maginot, 21 divisions de forteresse se trouvèrent enfermées dans un abri magnifique mais statique, dénuées de mobilité, inaptes à la manœuvre, à l'inverse de septembre 1914. La concentration fut donc réalisée en quelques jours, un délai plus qu'honorable à l'horloge de 1914, mais inopérant au chronomètre de 1939. La France, à la remorque de la diplomatie britannique, avait déclaré la guerre à l'Allemagne le 3 septembre, trois jours après l'agression contre la Pologne. Trois semaines plus tard, la Pologne n'existait plus. Une timide offensive française dans la Sarre était arrêtée. Le front, si l'on peut employer ce terme, entrait en léthargie. Il fallait être alors pour le moins agrégé de casuistique pour interpréter le décret du 9 octobre ainsi publié au Journal officiel:

«Article $1^{\text {er }}$ : À la date du 10 octobre, le ministre de la Guerre remet l'exploitation des chemins de fer au ministre des Travaux publics et des Transports.

Article 2 : Le ministre des Travaux publics et des Transports met à la disposition du général en chef le réseau ferré de la zone des armées.

Article 3 : Le ministre des Travaux publics et des Transports donne au ministre de la Guerre délégation permanente ou temporaire pour l'exécution des transports militaires sur le réseau de l'intérieur. "

Par ailleurs, " en s'appuyant sur l'expérience des deux premiers mois de guerre ", le décret du 24 novembre 1939 retranchait douze départements de la zone des armées. Le gouvernement renvoyait vers l'intérieur des dizaines de milliers de permissionnaires, ce qui devait engendrer de sérieuses difficultés lors de leur rappel sur le front en mai 1940. La France, décidément, s'installait dans la drôle de guerre, cette surprenante conception politico-stratégique.

$\mathrm{Au}$ cours de cette période tragi-comique caricaturée par les chansons de Maurice Chevalier, la SNCF a joué son rôle et assumé sa mission du transport de concentration dans des conditions très satisfaisantes. Edouard Daladier l'a d'ailleurs reconnu dans son "Adresse » du 30 septembre aux cheminots, ainsi que le généralissime Weygand, successeur de Gamelin, dans un ordre du jour du 31 mai $1940^{22}$ (fig. 6a et 6b). Le service régulateur fut l'un de ceux, bien rares hélas, qui ne causa au commandement aucune déception. L'esprit " cheminot ", si vivace et si militaire en certaines circonstances, contribua grandement au succès de cette complexe organisation. La Société nationale pouvait-elle faire plus ? Comme le souligne François Caron dans la conclusion de son ouvrage : "L'expérience de l'entre-deux-guerres montrait que seule la modernisation du réseau permettait de répondre à la concurrence des autres moyens de transport $^{23}$. » La SNCF manqua du temps nécessaire pour atteindre ses objectifs.

22- SHD, Série 1919-1939, 7N4082 et 27N100.

23- CARON, 2005, p. 977. 


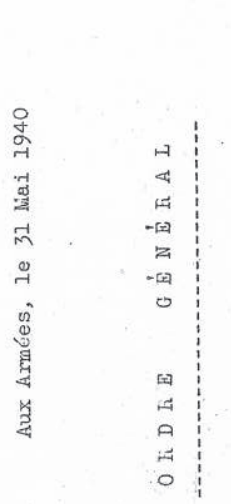

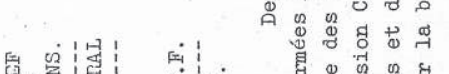
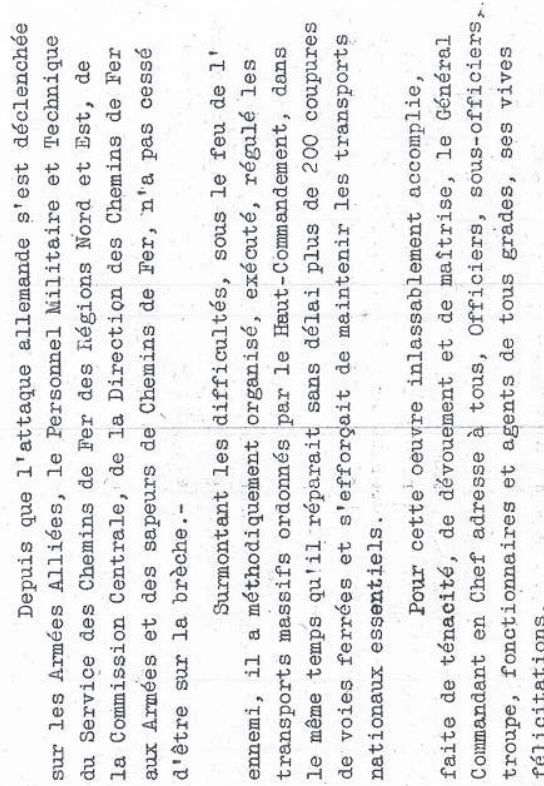

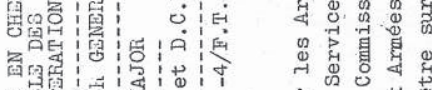

터ำ
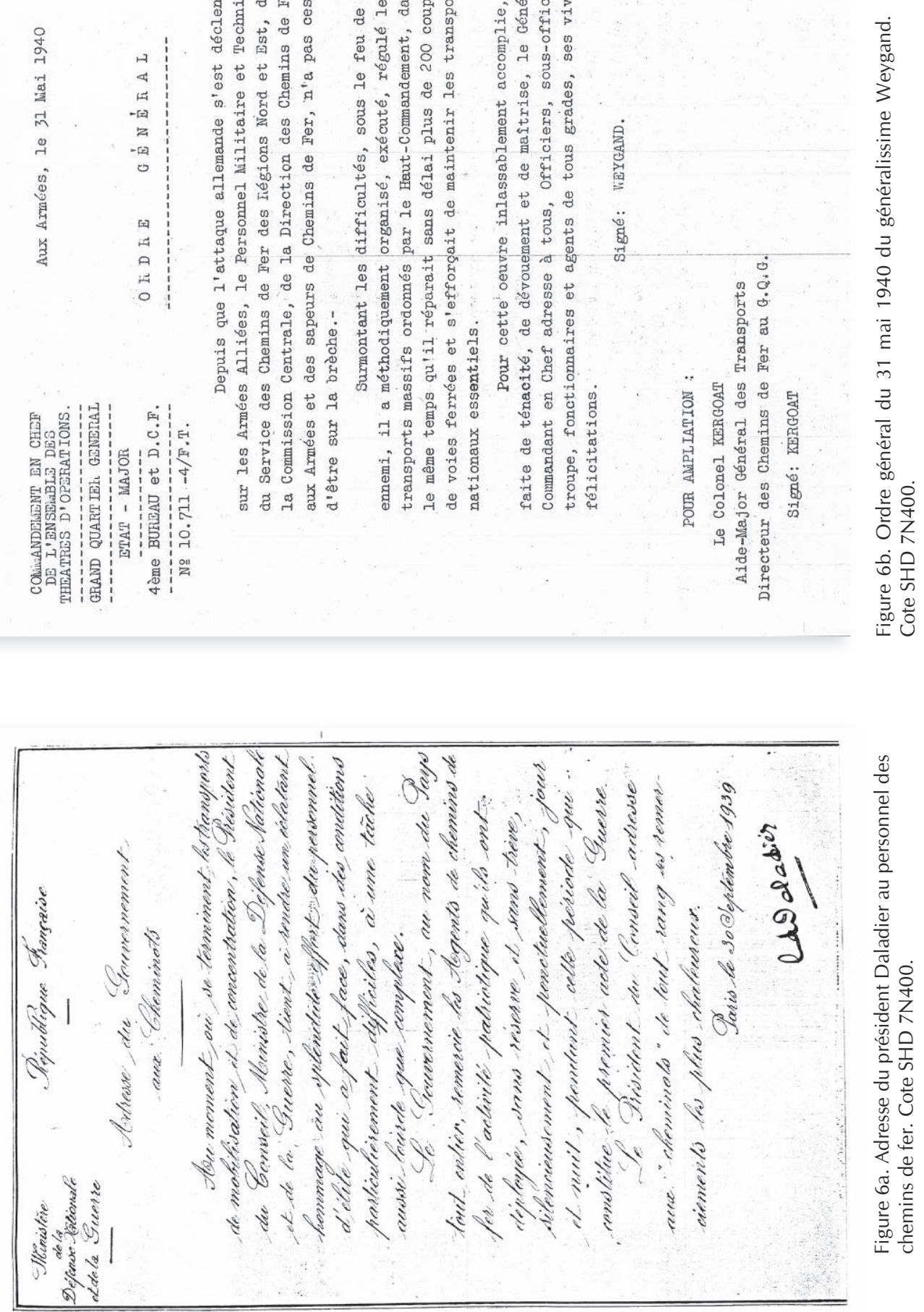
Mais à notre époque où la fonction militaire du rail n'est plus qu'un souvenir, le débat est purement rétrospectif, car à partir de la mi-mai 1940 la SNCF devint la cible d'une nouvelle dimension introduite dans l'espace vectoriel que nous évoquions plus haut : la suprématie aérienne. Les gouvernements faibles, handicapés par les crises ministérielles à répétition, comme les cerveaux de l'État-major dépassés dans leur réflexion stratégique, n’avaient imposé ni la vigilance, ni la réactivité, ni l'effort d'une énorme production industrielle, ces mesures indispensables pour faire face à la montée des périls. Le drame du printemps de 1940 résulte de la conjonction de toutes ces défaillances. Et, hélas, il n'y eut cette fois, aucun « miracle de la Marne ».

\section{Sources et bibliographie}

\section{Archives}

Archives du Service historique de la Défense

- Collection Campet de Saujon des ordonnances, lois, et des décrets militaires. De 1X1213 à 1X1245 pour 1913 et 1914. De 1X1868 à 1X1892 pour 1939 et 1940. Pour 1914-1918:

- Procès-verbaux du Conseil supérieur de la Guerre : 1N10-1N11 ; Conseil national économique 2N148 ; Procès-verbaux du Conseil supérieur de la Défense nationale série 7N ; 7N2042 à $2045 ; 16$ N2808-2811; 16N28132815-2816-2821-2824; 16N2820; 16N2863. Fonds Joffre : 14N1; 14N16. Fonds Buat : 6N18.

- Annuaire de l'Armée française 1914.

Pour 1919-1940:

- Cartons : 2N148; 7N 4050, 4060, 4064, 4071, 4072, 4073, 4077 à 4079, $4082 ; 27 \mathrm{~N} 100-102,7 \mathrm{~N}$ 409-411-2047.

Le carton 7-N-2047 est particulièrement intéressant. Il comporte un historique complet du $4^{\text {e }}$ Bureau de l'État-major des Armées pour la période 1939-1940, reconstitué par ses anciens officiers sous la direction des généraux Clément Blanc et Boyer.

- Annuaire de l'Armée française 1939.

Archives départementales

Série $S$ (travaux publics)

- Meuse : 40 S 507 ; 55 Sp 26 ; 56 S 1; 59 S 95; 60 S 607 ; 39 S 16 ; 40 S $540 ; 59$ S 12.

- Moselle : 7 S 3-14-18-19-60-65-66. 10 S 161-162-240. 


\section{Sources imprimées}

Collections consultées aux Archives de I'Assemblée nationale

- Jean-Baptiste Duvergier, Collection des Lois, décrets, règlements et avis du Conseil d'État. Années 1877, 1888, 1914.

- Secrétariat général de la présidence, Recueil des Lois d’intérêt général, Années 1914-1939.

- Annales de la Chambre des députés, Débats Troisième République.

- Collections du Journal officiel, du 4 septembre 1870 à 1940.

Ouvrages

GRANDMAISON, 1911 : Lieutenant-Colonel Loyseau de Grandmaison, Louis, [1861-1915], Deux conférences aux officiers du Centre des hautes études militaires (CHEM), Paris, Berger-Levrault, 1911.

LE HÉNAFF, 1922a : Colonel Le Hénaff, Joseph-Hippolyte-Félix et capitaine Bornecque, Henri, Les Chemins de fer français et la guerre, Paris, Librairie Chapelot, 1922.

LE HÉNAFF, 1922b : Colonel Le Hénaff, Joseph-Hippolyte-Félix, « La préparation et l'exécution d'un plan de transports de concentration. Les chemins de fer et le choix des zones les plus favorables à une action offensive ", Revue militaire française, Tome IV, nouvelle série (1922, avril-juin).

LE HÉNAFF, 1923 : Général Le Hénaff, Joseph-Hippolyte-Félix, Le Rôle militaire des chemins de fer, préface du général Raguenau, Paris, BergerLevrault, 1923.

PESCHAUD, 1926 : Peschaud, Marcel, La Guerre et les transports, Paris, PUF, 1926.

PESCHAUD, 1927 : Peschaud, Marcel, Les Chemins de fer allemands et la guerre, Paris, Lavauzelle, 1927.

PIERREFEU, 1922 : de Pierrefeu, Jean, GQG, Secteur 1, Paris, Éditions G. Grès, 1922, 2 vol., 304 p. et 259 p.

ROBIDA, s.d. : Capitaine Robida, "Souvenirs d'un officier régulateur des chemins de fer 1939-1940», SHD, Vincennes 27N100.

\section{Bibliographie}

BAUDOUİ, 1992 : Baudouï, Rémi, Raoul Dautry, le technocrate de la République, Paris, Balland, 1992.

CARON, 2005 : Caron, François, Histoire des chemins de fer en France, tome deuxième, 1883-1937, Paris, Fayard, 2005. 
CARON et CARDOT, 1991 : Caron, François, Cardot, Fabienne (sous la dir.), Histoire de l'électricité en France, tome premier, Espoir et conquêtes, 1881-1918, Paris, Fayard, 1991 ; LÉVY-LEBOYER, Maurice, et MORSEL, Henri (sous la dir.), tome deuxième, Linterconnexion et le marché, 1919-1946, ibid., 1994 ; MORSEL, Henri (dir.), tome troisième, Une cuvre nationale : l'équipement, la croissance de la demande, le nucléaire, 1946-1987, ibid., 1996.

DOISE et VAÏSSE, 1987 : Doise, Jean, et Vaîsse, Maurice, Diplomatie et outil militaire. Politique étrangère de la France : 1871-1969, Paris, Imprimerie Nationale, coll. "Politique étrangère de la France ", 1987 (nouvelle éd. : Diplomatie et outil militaire. Politique étrangère de la France : 1871-1991, Le Seuil, coll. " Points Histoire ", 1992).

GERNIGON, 1999 : Gernigon, Alain, Histoire de la signalisation ferroviaire française, Paris, La vie du rail, 1999.

LEPAGE, 1994 : Lepage, Pierre, "L'électrification des chemins de fer du Nord-Est ", in "Électricité, armement, défense ", Bulletin de l'AHEF, $\mathrm{n}^{\circ} 23$ (juin 1994), p. 19-34.

LEPAGE, 1996 : Lepage, Pierre, " De la mobilisation à la concentration. Le plan de transport français en août 1914 ", in "Armées et chemins de fer en France ", Revue d'histoire des chemins de fer, no 15 (automne 1996), p. 73-87.

LEPAGE, 1997 : Lepage, Pierre, "L'électrification des chemins de fer français. De Clemenceau à Pétain, le déclin de la volonté ", in "Électricité et chemins de fer, cent ans de progrès ferroviaire en France par l'Électricité ", Actes du colloque organisé par l'AHICF et par l'Association pour l'histoire de l'électricité en France à Paris, 17-19 mai 1995, Revue d'histoire des chemins de fer hors série $\mathrm{n}^{\circ} 5$ /collection Histoire de l'électricité $\mathrm{n}^{\circ} 10$, Paris, PUF, 1997, p. 167-186.

PÉDRONCINI, 1977 : Pédroncini, Guy, « Stratégie et relations internationales : la séance du 9 janvier 1912 au Conseil supérieur de la Défense nationale ", Revue d'histoire diplomatique, XCI (janvier-juin 1977), p. 143158.

SCHONTZ, FELTEU et GOURLOT, 1999 : Schontz, André, Felteu, Arsène, Gourlot, Marcel, Le Chemin de fer en Lorraine, préface de François Roth, Metz, Éditions La Serpenoise, 1999.

YVERT, 2002 : Yvert, Benoît (sous la dir.), Premiers ministres et présidents du Conseil depuis 1815. Histoire et dictionnaire raisonné, Paris, Perrin, 2002. 


\section{Annexes}

\section{Principaux textes législatifs et réglementaires auxquels il est fait référence, publiés ou non au Journal officiel suivant leur caractère de secret militaire}

- Loi du 3 juillet 1877 et décret du 2 août 1877 plaçant les chemins de fer sous la tutelle du ministère de la Guerre en cas de guerre.

- Loi du 28 décembre 1888 portant réquisition des chemins de fer en temps de guerre.

- Loi du 8 août 1890 portant déclaration d'utilité publique le doublement de la ligne de Paris à Avricourt entre Vitry-le-François et Lérouville.

- Décret du 2 décembre 1913 portant règlement sur les armées en campagne. (En particulier article 8 et annexe 18 relatifs au chemin de fer.)

- Décret du 8 décembre 1913 portant règlement sur les transports stratégiques par chemin de fer.

- Arrêté ministériel du 2 août 1914 portant affectation de l'ensemble des réseaux de chemins de fer aux besoins militaires.

- Arrêté ministériel du 2 août 1914 délimitant la zone des armées ainsi que les réseaux de communications placés sous l'autorité du général commandant en chef, et suspendant les services commerciaux.

- Décret du $1^{\text {er }}$ août 1914 portant mobilisation des armées de terre et de mer.

- Décret du 5 août 1914 déclarant l'état de siège sur les départements métropolitains et l'Algérie.

- Arrêté du 19 juin 1919 relatif à l'organisation du réseau ferré d'Alsace-Lorraine.

- Décret du 30 novembre 1920 portant rattachement des chemins de fer d'Alsace-Lorraine au ministère des Travaux publics.

- Loi du 28 février 1923 déclarant d'utilité publique la création d'une nouvelle ligne de Lérouville à Metz.

- Loi du 30 mars 1923 portant rectification du décret du 30 novembre 1920 relatif au rattachement du réseau d'Alsace-Lorraine au ministère des Travaux publics.

- Décision ministérielle du 6 février 1928 déclarant l’urgence de l'agrandissement de la gare de Lérouville.

- Loi du 7 mars et décret du 1929 déclarant d'utilité publique la création du saut de mouton de Xouaxange.

- Décret du 31 août 1937 portant réorganisation du régime des chemins de fer.

- Décret du 28 décembre 1937 portant création de la SNCF.

- Décret du 17 avril 1938 portant organisation des services de transports en temps de guerre. 
- Décret du 25 avril 1938 portant réquisition des voies ferrées exploitées par la SNCF pour les besoins militaires.

- Loi du 11 juillet 1938 sur l'organisation de la nation pour l'état de guerre.

- Décret du 28 novembre 1938 portant réquisition des agents et ouvriers des services publics de l'Etat, des départements, des communes et des services concédés.

- Décret du 4 février 1939 modifiant la composition de la Commission supérieure des chemins de fer.

- Décret du 29 juillet 1939 relatif au programme de premier établissement de la SNCF.

- Arrêté ministériel du 24 août 1939 portant réquisition des ressources des compagnies de chemins de fer pour les besoins militaires et suspendant les transports commerciaux.

* Décret du $1^{\text {er }}$ septembre 1939 : mobilisation générale des armées de terre, de mer et de l'air.

- Décret du $1^{\text {er }}$ septembre 1939 délimitant la zone des armées.

- Arrêté ministériel du $1^{\mathrm{er}}$ septembre 1939 déclarant la France en état de siège.

- Décret du 4 septembre 1939 déclarant l'état de guerre avec l'Allemagne.

- Arrêté interministériel du 9 octobre 1939 redéfinissant le régime de l'exploitation des chemins de fer pendant la période d'opérations.

- Voir aussi SHD 7N4050, $7^{\text {e }}$ conférence de 1924 au CSG, «Le réseau ferré stratégique ", par le Lieutenant-colonel Lamson, chef du $4^{e}$ Bureau de l'EMA, Secret, 26 feuillets. 
II. Les principaux interlocuteurs des chemins de fer militarisés

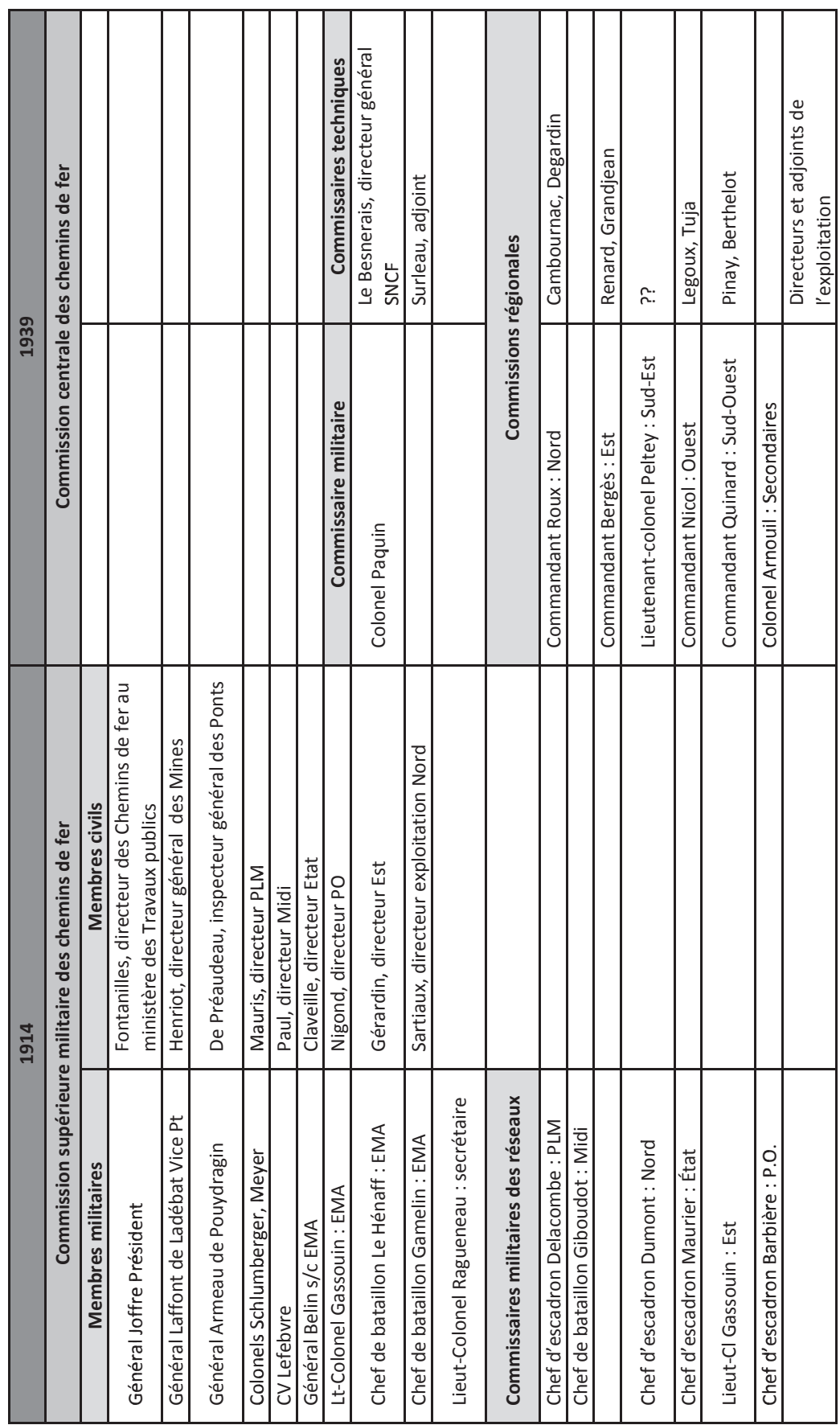


